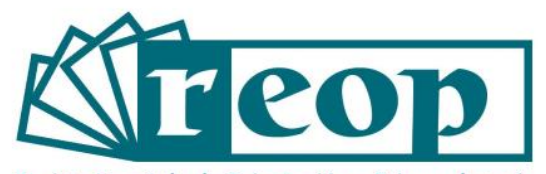

Revista Española de Orientación y Psicopedagogía

\title{
¿EL BAJO RENDIMIENTO ACADÉMICO MEJORA A PARTIR DE LA INTERVENCIÓN COGNITIVA COMPUTARIZADA?'1
}

\section{DOES LOW ACADEMIC PERFORMANCE IMPROVE WITH COMPUTERIZED COGNITIVE INTERVENTION?}

\author{
Ángela Pilar Albarracín Rodríguez ${ }^{2}$ \\ Universidad Pontificia Bolivariana seccional Bucaramanga. Facultad de Psicología. Escuela de \\ Ciencias Sociales. Bucaramanga, Colombia \\ David Andrés Montoya Arenas \\ Universidad Pontificia Bolivariana. Facultad de Psicología. Escuela de Ciencias Sociales y \\ Universidad de San Buenaventura Medellín, Doctorado en Psicología. Medellín, Colombia
}

\section{RESUMEN}

El bajo rendimiento académico influye en la repetición y deserción escolar afectando a la persona, su familia y la institución educativa siendo primordial proporcionar estrategias que favorezcan el logro académico y la permanencia de los estudiantes en colegios y universidades. El objetivo general de este estudio fue determinar la efectividad de un programa de intervención computarizado en funciones ejecutivas (ACTÍVATE+) diseñado para estudiantes con bajo rendimiento académico. Esta investigación tuvo un diseño cuasiexperimental pre-post con grupo control, se contó con una muestra de 104 estudiantes entre los 13 y 19 años que conformaron los dos grupos, un grupo experimental $(n=46)$ y un grupo control $(n=58)$. Ambos grupos recibieron sesiones de apoyo psicopedagógico y el grupo experimental recibió además el programa de intervención ACTÍVATE+. Asimismo, se aplicaron pruebas neuropsicológicas y se registró el rendimiento académico antes y después de la intervención. Los resultados evidenciaron que existen diferencias en el desempeño ejecutivo y rendimiento académico entre los grupos control y

${ }^{1}$ Fuente de financiación: Universidad Pontificia Bolivariana seccional Bucaramanga y Universidad de San Buenaventura Medellín.

${ }^{2}$ Correspondencia: Angela Pilar Albarracín Rodríguez. Correo-e: angela.albarracin@upb.edu.co 
experimental después de la intervención. En conclusión, el programa ACTíVATE+ se constituye en una herramienta eficaz en el entrenamiento de las funciones ejecutivas influyendo positivamente en el incremento del rendimiento académico de estudiantes con bajo desempeño escolar, tornándose en un instrumento de apoyo para los profesionales que orientan procesos psicopedagógicos en las instituciones educativas.

Palabras clave: psicología del desarrollo, rendimiento académico, aplicación informática, estudiantes, funciones ejecutivas.

\begin{abstract}
Low academic performance influences students' retention and school dropout, affecting the person, the family, and the educational institution. It is thus essential to provide strategies that promote academic achievement and avoid dropping out of school. The objective was to determine the effectiveness of a computerized intervention program in executive functions (ACTIVATE+) designed for students with low academic performance. This research had a pre-post quasiexperimental design with a control group, the sample was comprised of 104 students between 13 and 19 years old distributed in two groups (experimental group $n=46$ and control group $n=58$ ), both of which received psychopedagogical support, and the experimental group also received the ACTIVATE + intervention program. Likewise, neuropsychological tests were administered, and academic performance was recorded before and after the intervention. The results showed that there are differences in executive performance and academic performance between the control and experimental groups after the intervention. In conclusion, the ACTÍVATE + program is an effective tool in the training of executive functions, positively influencing the improvement of the academic performance of students with low school performance, becoming a support instrument for professionals who deliver psychopedagogical guidance in educational institutions.
\end{abstract}

Key Words: developmental psychology, academic achievement, computer application, executive functions, students.

\title{
Cómo citar este artículo:
}

Albarracín, A. y Montoya, D. (2021). ¿El bajo rendimiento académico mejora a partir de la intervención cognitiva computarizada? Revista Española de Orientación y Psicopedagogía, 32(3), 74-92. https://doi.org/10.5944/reop.vol.32.num.3.2021.32558 


\section{Introducción}

El bajo rendimiento académico es una de las primeras razones por la cual los estudiantes pueden presentar deserción y/o repetición escolar siendo las causas diversas y variadas asociadas a factores individuales, familiares, del sistema educativo, regionales y contextuales (Torres et al., 2015; Korhonen et al., 2014). De acuerdo con la OEI (2010) en América Latina y el Caribe se estima que entre el $40 \%$ y $60 \%$ de los jóvenes no alcanzan niveles básicos de formación. Asimismo, se encuentra que menos del $70 \%$ de los estudiantes llegan al último grado de secundaria y el $29 \%$ han repetido al menos un año (Sánchez et al., 2016). En Colombia a nivel de educación superior se ha encontrado una tasa de deserción entre el $40 \%$ y 50\% mostrando que uno de cada dos estudiantes que ingresan a la universidad no finaliza sus estudios (SPADIES, 2017) presentando una deserción hasta del 35\% en los primeros cuatro semestres (SPADIES, 2017).

Lo anterior evidencia la necesidad de establecer mecanismos académicos y administrativos que controlen estos fenómenos (MEN, 2009) con el fin de aportar a la cobertura de la educación y permanencia de los estudiantes con herramientas que sirvan de apoyo a los profesionales que orientan estos procesos. A nivel general se encuentra que las estrategias o programas de intervención focalizados a la captación y retención de estudiantes de colegios están dirigidos al refuerzo académico, la acción tutorial, la intervención familiar, las habilidades sociales y las técnicas de estudio (Miñaca y Hervás, 2013); y a nivel universitario se han orientado a estrategias de autorregulación, tutorías y áreas específicas como los valores y comprensión lectora (Albarracín y Montoya, 2016). A pesar de contar con evidencias de la relación e influencia de las funciones ejecutivas (FE) sobre el desempeño académico demostrando que estas pueden ser predictores de éxito académico interviniendo en habilidades como el cálculo y la lectura (Knouse et al., 2014; Roebers et al., 2012), la intervención cognitiva y particularmente de las FE para mejorar el desempeño académico se ha realizado en menor medida (Albarracín y Montoya, 2016).

Estudios sobre la relación entre FE y bajo rendimiento académico en adolescentes (Rojas y Rincón, 2015) y jóvenes universitarios (Barceló et al., 2006) han planteado que, algunas de las causas del bajo rendimiento académico podrían ser el poco desarrollo de habilidades cognoscitivas o el déficit en las mismas, es decir dificultades en las FE. Del mismo modo, Rodríguez (2016) encontró en un grupo de estudiantes con bajo rendimiento académico dificultades en fluidez verbal, fluidez gráfica y flexibilidad cognitiva y un menor desarrollo en procesos como planeación y organización en comparación con el grupo de alto rendimiento, afirmando que las FE son susceptibles de mejorar con programas de intervención sólidos. Al mismo tiempo, las FE parecen ser relativamente independientes de las mediciones de cociente intelectual $(\mathrm{Cl})$ según el estudio de Montoya et al. (2018) y los resultados obtenidos en el examen de estado SABER PRO encontraron que la alta capacidad intelectual no determinaba un desempeño alto en la prueba, ni garantiza un adecuado rendimiento académico (Bahamón y Reyes, 2014). Lo anterior muestra que el entrenamiento en FE puede ser una oportunidad para favorecer el desempeño académico de los escolares.

En este punto, es preciso señalar que el periodo de la adolescencia es una etapa crítica y fundamental en el desarrollo de las funciones cerebrales (frontales y ejecutivas) donde los períodos de mayor rapidez de desarrollo se han relacionado con las etapas de escolarización. Por tanto, se ha planteado que procesos como la flexibilidad cognitiva, la memoria de trabajo y la resolución de problemas complejos pueden seguir su maduración hasta los 19 años (García, González et al., 2014) y funciones como la planeación secuencial, fluidez verbal y actitud abstracta pueden desarrollarse hasta los 30 años siempre que la persona se encuentre en contextos escolarizados (Flores et al., 2014). 
En este sentido, investigaciones como la de Holmes y Gathercole (2014) quienes aplicaron un programa de intervención en memoria de trabajo para niños con bajo rendimiento académico evidenció resultados positivos; al igual que el estudio desarrollado por Bernal-Ruiz et al. (2020) encontrando resultados favorables en el desempeño del control inhibitorio en niños entre los 6 y 7 años después de intervenir en las FE. No obstante, este tipo de estudios se han conducido principalmente en población infantil (Arán-Filippetti y Richaud de Minzi, 2011; García, Rodríguez et al., 2013; Holmes y Gathercole, 2014); en tanto que en población universitaria se han dirigido al aprendizaje autorregulado (Núñez et al. 2011) y al aprendizaje estratégico (Carbonero et al., 2013) lo que demuestra que la aplicación de la neuropsicología en el campo educativo es todavía incipiente (Pérez, 2015, Montes et al., 2020), considerándose importante continuar con el desarrollo de intervenciones en procesos como las FE con el fin de contribuir a mejorar el desempeño académico de los estudiantes. Igualmente, diversos autores proponen la inclusión de tecnologías de la información y comunicación (TIC) para lograr un mayor impacto y efectividad en las intervenciones (Benítez et al., 2014; Núñez et al., 2011), además de generar entornos más flexibles (Cabero, 2010; Álvarez, 2012) y mayor motivación en los participantes (Núñez et al. 2011).

En resumen, se evidencia la pertinencia de contar con programas de intervención en FE enfocados a mejorar el rendimiento académico de los estudiantes dada la escasez de este tipo de programas y a la necesidad actual de generar estrategias de intervención y acompañamiento psicopedagógico que empleen las TIC (Albarracín y Montoya, 2016, Benítez et al., 2014; Núñez et al., 2011) teniendo en cuenta la inclusión de estas tecnologías en los procesos educativos, siendo una alternativa no invasiva de entrenamiento cognitivo (Blanco et al., 2020). Además, se considera que este campo se encuentra en un estado investigativo inicial siendo necesario incrementar estudios experimentales que permitan llegar a conclusiones más concretas y que fortalezcan este campo de investigación (Montes et al., 2020) con el fin de contar con herramientas pertinentes para los procesos psicopedagógicos en la población adolescente con bajo rendimiento académico.

Por consiguiente, este estudio tuvo como propósito determinar la efectividad del programa de intervención ACTÍVATE+ en FE diseñado para estudiantes con bajo rendimiento académico con el fin de favorecer el logro del éxito académico y como apoyo a los profesionales y programas psicopedagógicos de las instituciones educativas para prevenir la repetición escolar e incrementar la permanencia de los estudiantes.

\section{Método}

El diseño de este estudio fue cuasiexperimental pre-post con grupo control (Montero y León, 2005). Se conformaron dos grupos: 1) uno experimental al que se le aplicó el programa ACTÍVATE + y las sesiones de apoyo psicopedagógico; 2) y uno control al que se le aplicaron solo las sesiones de apoyo psicopedagógico. 


\section{Muestra}

La muestra se seleccionó de forma no probabilística por criterio. Se incluyeron estudiantes entre los 13 y 19 años $(M=16,38 ; D E=1,62)$ con bajo rendimiento académico (promedio igual o inferior a 3,5 y pérdida o cancelación de mínimo un curso) todos pertenecientes a dos instituciones del departamento de Antioquia y una institución del departamento de Santander que registraron de manera completa las sesiones del programa ACTíVATE+. La asignación a los grupos no se realizó de forma aleatoria en la muestra perteneciente a Antioquia y se excluyeron 10 estudiantes que presentaron un $\mathrm{Cl}$ inferior a $79 \mathrm{y} / 0$ algún trastorno de aprendizaje, neurológico 0 neuropsiquiátrico. La muestra quedó conformada por 104 estudiantes, 58 (55,8\%) pertenecientes al grupo control y $46(44,2 \%)$ al grupo experimental, asimismo, el 52,9\% eran del género masculino y el $47,1 \%$ del género femenino.

\section{Instrumentos y/o técnicas}

Para el registro de datos sociodemográficos y verificación de los criterios de inclusión - exclusión se emplearon: La entrevista sociodemográfica, la MINI Entrevista Neuropsiquiátrica Internacional MINI-KID (Shehan et al., 2006, adaptación al español colombiano por Pineda, 2006) o MINI adultos (Ferrando et al., 2000) y la prueba Breve de Inteligencia de Kaufman (K-BIT, Kaufman y Kaufman, 1997).

Para la evaluación pretest y postest de las FE se aplicaron las pruebas:

Trail Making Test (TMT A y B) esta prueba evalúa la planeación o planificación, velocidad en la búsqueda visual, secuenciación visoespacial, atención, flexibilidad mental y función ejecutiva (Lezak, 1995; Spreen y Strauss, 1998; Tombaugh, 2004). Su duración es de 10 minutos aproximadamente y se registran aciertos, omisiones, tiempo de ejecución y uniones erradas. En población colombiana ha demostrado una fiabilidad de 0,7 (Arango et al., 2017; Arango y Rivera, 2015).

Test de Clasificación de Tarjetas Wisconsin (WCST, Fortuny y Heaton, 1996) esta prueba mide la capacidad de resolución de problemas, planeación y modificación de estrategias cognitivas ante los cambios del ambiente (Pineda, 2000). Se empleó la versión computarizada de Fortuny y Heaton (1996) y tiene una duración de 15 minutos aproximadamente. Ha demostrado una fiabilidad de 0,57 . Para la evaluación postest se realizó cambio en la secuencia de las categorías, por ejemplo, si en el pretest se inició con las categorías $\mathrm{F}$ (forma), $\mathrm{N}$ (número) y $\mathrm{C}$ (color) para el postest se empleó la secuencia C, F y N.

Test de palabras y colores Stroop (Golden, 2010) esta prueba examina la respuesta inhibitoria de las FE (Arango y Rivera, 2015) y su administración es de 5 minutos aproximadamente (Golden, 2010). El Stroop en población colombiana obtuvo un coeficiente de fiabilidad ponderado de 0,85 para Lectura de Palabras, 0,86 para Nominación de Colores y 0,71 para Interferencia (Arango y Rivera, 2015).

Para la evaluación de la memoria de trabajo se emplearon las tareas de las Escalas Wechsler (Wechsler, 2002, 2005) Dígitos, Letras y Números, y Aritmética descritas a continuación:

Dígitos directos e inversos: Este subtest evalúa la memoria auditiva a corto plazo, capacidad de seguir una secuencia, atención y concentración. Específicamente, Dígitos Directos permite la evaluación de la capacidad de aprendizaje, codificación y procesamiento auditivo; mientras que 
Dígitos Inversos mide la memoria de trabajo, transformación de la información e imaginación visoespacial. Para el postest se realizó cambio de las secuencias de los números tanto directos como inversos.

Letras y Números: Esta tarea presenta series de combinaciones de letras y números de manera desordenada, donde el evaluado debe reproducir de manera ordenada cada serie, los números en orden ascendente y las letras en orden alfabético. Este subtest evalúa el manejo de información mental, formación de secuencias e imaginación visoespacial, memoria auditiva a corto plazo, atención y velocidad de procesamiento. Para el postest se cambiaron las secuencias de las letras y los números.

Aritmética: Esta tarea consiste en la presentación verbal de 20 problemas que deben ser resueltos mentalmente por el evaluado y mide el manejo mental de la información, concentración, atención, memoria a corto y largo plazo, razonamiento numérico, alerta mental y disminución de la influencia de los conocimientos matemáticos y mayor carga de memoria de trabajo.

Test de Fluidez Verbal Semántica y Fonológica (FVS - FVF, Borkowski et al., 1967) este test mide la capacidad de producir palabras comenzando por una letra determinada en un tiempo específico. Para este trabajo se aplicó la Fluidez Verbal Fonológica (FVF) que evalúa específicamente FE y consiste en que la persona debe generar la mayor cantidad de palabras que comiencen por el sonido de las letras F, A, S y M, 60 segundos por cada letra (Arango y Rivera, 2015). La prueba genera la puntuación total por cada letra tiene una duración de 5 minutos aproximadamente y presenta un coeficiente de fiabilidad ponderado de 0,82 (Arango y Rivera, 2015). Para el postest se cambió la secuencia de las letras, por ejemplo, si en el pretest se empleó la secuencia FASM, en el postest se utilizó la secuencia MSAF.

Rendimiento académico: Se tuvo en cuenta la calificación final por asignaturas y el promedio general del estudiante en todo el periodo académico antes de la intervención y el último periodo académico después de la intervención. Las calificaciones en las instituciones educativas oscilaban de 0,0 a 5,0, siendo 5,0 la máxima calificación.

Sesiones de apoyo psicopedagógico: Consistió en brindar 18 sesiones extraescolares de asesoría psicológica a los estudiantes con algún tipo de dificultad académica en aspectos psicoemocionales asociados a los procesos de aprendizaje y orientación psicopedagógica en estilos de aprendizaje, revisión de hábitos y técnicas de estudio.

Programa de intervención computarizado ACTÍVATE+: Para el diseño y desarrollo del programa ACTÍVATE+ se tuvieron en cuenta los antecedentes revisados sobre FE y su relación con el rendimiento académico, conceptos relacionados con rehabilitación cognitiva, programas de intervención y consideraciones para su desarrollo. La metodología para su elaboración se basó en el enfoque de restauración, el cual interviene directamente sobre las funciones cognitivas a través de la estimulación y mejora de estas con el fin de que alcancen un rendimiento óptimo (Lubrini et al., 2009). Por tanto, el Programa ACTíVATE+ tiene como objetivo estimular las FE en estudiantes con bajo rendimiento académico para potenciar su funcionamiento cognitivo y así contribuir a la mejora de su desempeño académico. Se desarrollaron 72 tareas que entrenan cuatro FE: Control inhibitorio, Planeación, Memoria de Trabajo y Fluidez verbal (18 tareas por cada FE). Estas tareas son de duración breve, donde las actividades varían en complejidad, es decir, que en los primeros niveles se inicia con ejercicios sencillos y luego van aumentando gradualmente su dificultad en los niveles posteriores (Portellano y García, 2014). Por consiguiente, el Programa ACTíVATE+ está conformado por 18 sesiones, cada una compuesta por cuatro tareas (una por cada FE) que se aplican con una frecuencia de tres veces por semana y con una duración máxima de 30 minutos por sesión. El diseño del programa ACTíVATE+ tuvo una duración de dos años, es un software online implementado en plataforma web para uso en Google Chrome (Figura 1) cumpliendo los protocolos de almacenamiento seguro de la información. Este software está registrado ante el Ministerio del Interior de Colombia y cuenta con un manual de aplicación. 


\section{Figura 1}

Imágenes programa ACTíVATE+ (ingreso, selección sesiones, tarea)

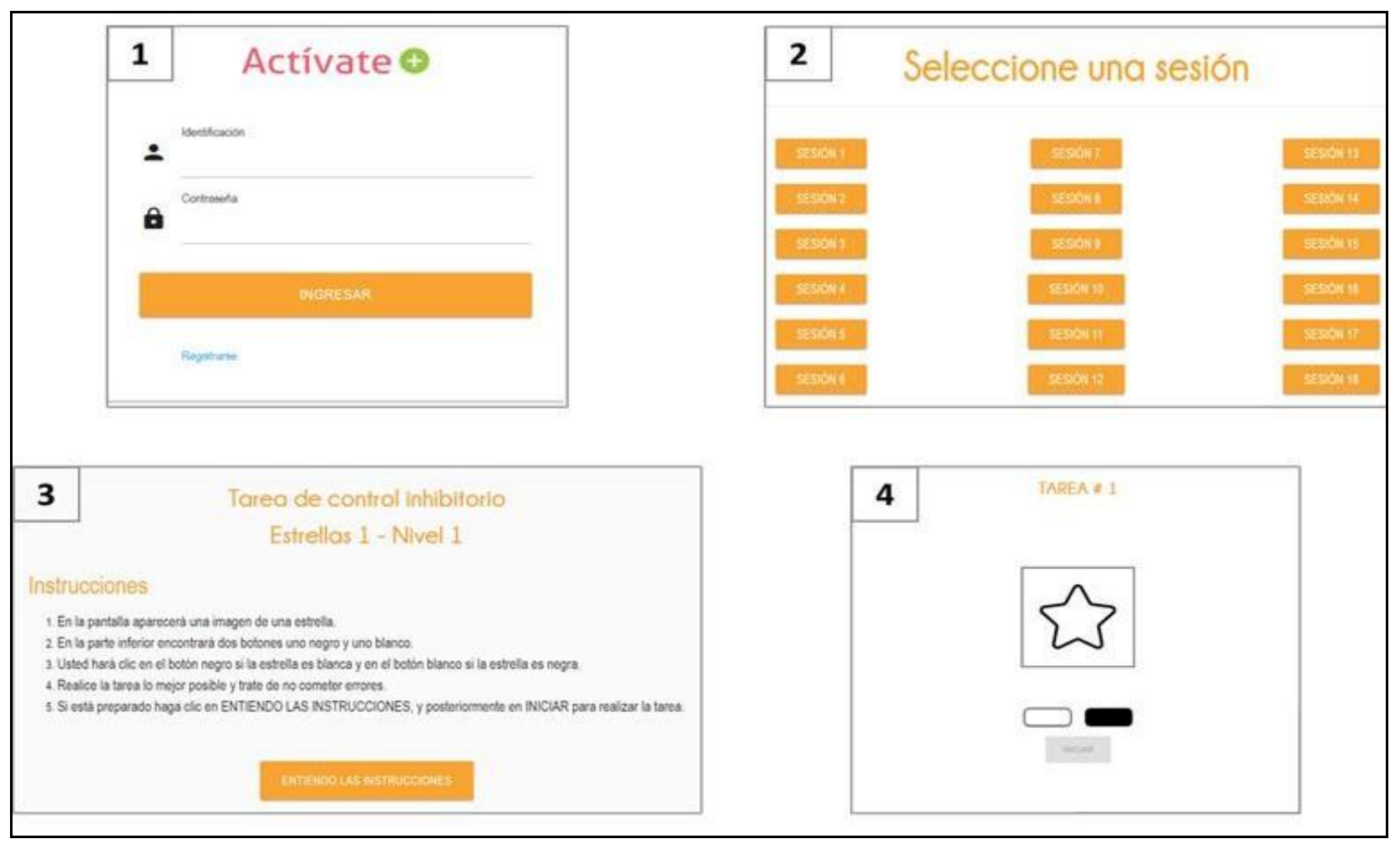

Fuente: Elaboración propia

En la figura 1 se observa que la imagen 1 presenta la página inicial para registro (por primera vez) o ingreso al programa, posteriormente se da una bienvenida y se pasa a la selección de las sesiones (imagen 2) allí cada sesión contiene cuatro tareas por cada FE. Al inicio de cada tarea se muestran las instrucciones (imagen 3), cuando el participante ha comprendido lo que debe realizar puede iniciar la tarea (imagen 4) y al finalizar el programa muestra una retroalimentación en función de aciertos, errores, tiempo empleado, esta información depende del tipo de tarea realizada. Finalizada una sesión, se puede seleccionar la siguiente.

\section{Procedimiento}

Primero, se realizó el diseño y desarrollo en formato computarizado del programa ACTíVATE+. Segundo, se presentó el proyecto a las instituciones educativas para su aprobación y se inició con la selección de la muestra según la edad y rendimiento académico. Tercero, se realizó contacto personal con los estudiantes seleccionados para informar acerca del proyecto y fueron incluidos previo diligenciamiento del consentimiento informado, según la edad del participante se solicitó autorización al representante legal garantizando el derecho a la confidencialidad y el anonimato. Después de obtener la autorización, se programó la primera sesión para la aplicación de la Entrevista Sociodemográfica, MINIKID o MINI adultos y K-BIT verificando criterios de inclusión en una sesión de una hora aproximadamente. Si el estudiante cumplía con los criterios, se procedía 
en otra sesión de una hora a la aplicación aleatoria pretest de las pruebas neuropsicológicas y el registro del rendimiento académico. Cuarto, se asignaron los estudiantes a uno de los dos grupos (experimental o control) procurando emparejar los grupos de acuerdo con la edad, género y escolaridad; asimismo, ambos grupos realizaron las sesiones de apoyo psicopedagógico de las instituciones educativas.

Para la aplicación del programa ACTÍVATE+ al grupo experimental, se dispuso de salas de computación con conexión a internet por cable y se realizaron las sesiones de forma colectiva (grupos de 4 personas y en tiempo extraescolar), el grupo de estudiantes siempre estuvo acompañado por un profesional para el acceso al programa y desarrollo de las sesiones en caso de dudas o inquietudes. Así mismo, el profesional contaba con el usuario y contraseña de cada participante con el fin que el estudiante no pudiese ingresar en otros momentos a las sesiones y tareas. Después de 6 semanas de intervención, se realizó la evaluación postest aplicando nuevamente los instrumentos de FE de forma aleatoria, con cambios de secuencia y se registró el rendimiento académico. El proceso fue ejecutado por dos estudiantes de maestría en neuropsicología y una candidata a doctorado en psicología bajo la supervisión de un doctor en neuropsicología y tuvo una duración de 6 meses aproximadamente.

Para el análisis estadístico de los datos se empleó el SPSS 25.0, con el cual se llevaron a cabo análisis univariados descriptivos de los datos de la muestra y de los puntajes pretest y postest de las pruebas neuropsicológicas y el rendimiento académico. Se emplearon pruebas no paramétricas Wilcoxon y U de Mann Whitney para comparar el desempeño de los participantes a nivel intra e intergrupal antes y después de la intervención. Finalmente, para medir la efectividad del programa se emplearon análisis de comparaciones no paramétricos Wilcoxon y $U$ de Mann Whitney del rendimiento académico antes y después de la intervención. Para las pruebas estadísticas se estableció como nivel de significancia $p<0,05$, además se obtuvo el tamaño del efecto $(r)$.

\section{Resultados}

A continuación, se presentan las características sociodemográficas como género, escolaridad, edad y cociente intelectual $(\mathrm{Cl})$ de cada uno de los grupos (Tabla 1).

Igualmente, se analizaron las diferencias entre los grupos control y experimental en cuanto a las variables de edad, $\mathrm{Cl}$ y escolaridad con análisis no paramétricos $\mathrm{U}$ de Mann Whitney, los cuales mostraron que no existen diferencias significativas para la edad $(U=1207 ; p=0,38)$, el $\mathrm{Cl}$ $(U=1040,5 ; p=0,054)$ ni para los años de escolaridad $(U=832,5 ; p=0,32)$.

Posteriormente, se realizó el análisis de las puntuaciones pretest y postest de las pruebas neuropsicológicas del grupo control empleando la prueba Wilcoxon, el cual evidenció diferencias estadísticamente significativas en el dominio de Planeación para las puntuaciones de TMTAErrores $(Z=-3,53 ; p=0,00 ; r=-0,46)$ y TMTB-Errores $(Z=-4,99 ; p=0,00 ; r=-0,65)$ en ambas la menor puntuación se presentó en el postest. En el dominio de Control Inhibitorio se observaron diferencias en las puntuaciones de Interferencia del Stroop $(Z=-2,34 ; p=0,01 ; r=-0,3)$ siendo menor la puntuación en el postest. En el dominio de Memoria de Trabajo las diferencias se encuentran en la puntuación de Número Inversos $(Z=-2,08 ; p=0,03 ; r=0,27)$ siendo mayor en el postest y en Fluidez Verbal en la Letra $A(Z=-3,29 ; p=0,01 ; r=0,43)$ con una mayor puntuación también en el postest. 
Tabla 1

Características sociodemográficas de la muestra

\begin{tabular}{|c|c|c|c|c|c|c|c|c|}
\hline \multirow[b]{2}{*}{ Variable } & \multicolumn{4}{|c|}{ Grupo control $(n=58)$} & \multicolumn{4}{|c|}{ Grupo experimental $(n=46)$} \\
\hline & \multicolumn{2}{|c|}{ Categorías } & Frecuencia & $\%$ & \multicolumn{2}{|c|}{ Categorías } & Frecuencia & $\%$ \\
\hline \multirow{2}{*}{ Género } & \multicolumn{2}{|c|}{ Masculino } & 27 & 46,6 & \multicolumn{2}{|c|}{ Masculino } & 28 & 60,9 \\
\hline & \multicolumn{2}{|c|}{ Femenino } & 31 & 56,4 & \multicolumn{2}{|c|}{ Femenino } & 18 & 39,1 \\
\hline \multirow{3}{*}{ Escolaridad } & \multirow{2}{*}{\multicolumn{2}{|c|}{$\begin{array}{l}\text { hasta } 11 \text { años } \\
\text { más de } 11 \text { años }\end{array}$}} & 48 & 82,8 & \multirow{2}{*}{\multicolumn{2}{|c|}{$\begin{array}{l}\text { hasta } 11 \text { años } \\
\text { más de } 11 \text { años }\end{array}$}} & 32 & 69,6 \\
\hline & & & 10 & 17,2 & & & 14 & 30,4 \\
\hline & Mín. & Máx. & Media & DE & Mín. & Máx. & Media & DE \\
\hline Edad & 13 & 19 & 16,45 & 1,64 & 13 & 19 & 16,28 & 1,61 \\
\hline \multirow[t]{2}{*}{$\mathrm{Cl}$} & 79 & 118 & 106,29 & 10,65 & 84 & 129 & 109,61 & 11,04 \\
\hline & \multicolumn{2}{|c|}{ Mediana } & Q1 & Q3 & \multicolumn{2}{|c|}{ Mediana } & Q1 & Q3 \\
\hline Edad & \multicolumn{2}{|c|}{17} & 15 & 18 & \multicolumn{2}{|c|}{17} & 15,75 & 17 \\
\hline $\mathrm{Cl}$ & \multicolumn{2}{|c|}{110} & 102 & 114 & \multicolumn{2}{|c|}{113,5} & 100 & 117 \\
\hline
\end{tabular}

Seguidamente, el análisis de las puntuaciones pretest y postest de las pruebas neuropsicológicas por el grupo experimental utilizando la prueba Wilcoxon, mostró que existen diferencias estadísticamente significativas en la mayoría de las puntuaciones de todos los dominios de FE (Planeación, Memoria de Trabajo, Control Inhibitorio y Fluidez Verbal) en el postest. No se evidenciaron diferencias estadísticamente significativas para las puntuaciones de TMTB-Segundos $(p>0,05)$ ni WCST Aciertos $(p>0,05)$ del dominio de Planeación.

Al realizar la comparación pretest de los grupos control y experimental con la $\mathrm{U}$ de Mann Whitney, se encontró que, en los dominios de Planeación, Control Inhibitorio, Fluidez Verbal y Memoria de Trabajo, la mayoría de las puntuaciones fueron similares entre los grupos (Tabla 2). No obstante, se encontraron diferencias estadísticamente significativas para el dominio de Control Inhibitorio en la puntuación de Interferencia $(p=0,03)$, en Memoria de Trabajo para Dígitos Inversos $(p=0,006)$, Número Inversos $(p=0,000)$, Total Dígitos $(p=0,000)$, Aritmética $(p=0,001)$, Letras y Números $(p=0,01)$ y en Fluidez Verbal en la Letra $M(p=0,03)$ siendo las puntuaciones mayores en el grupo experimental antes de la intervención y mostrando un tamaño del efecto medio a bajo.

Igualmente, al analizar el desempeño de los grupos control y experimental con la $\mathrm{U}$ de Mann Whitney después de la intervención, los resultados revelaron diferencias estadísticamente significativas entre los grupos (Tabla 3), donde los participantes del grupo experimental demostraron un mejor desempeño que el grupo control en todos los dominios de FE $(p=0,000)$ exceptuando las puntuaciones de TMTB-Segundos $(p>0,05)$ y WCST Aciertos $(p>0,05)$ del dominio Planeación. Lo anterior sugiere que el entrenamiento en FE puede generar un impacto positivo en el incremento del desempeño ejecutivo. 
Tabla 2

Puntuaciones de las pruebas neuropsicológicas pretest grupos control y experimental

\begin{tabular}{|c|c|c|c|c|c|c|c|c|c|c|c|c|c|}
\hline \multicolumn{2}{|l|}{ Variable } & \multicolumn{3}{|c|}{$\begin{array}{c}\text { Grupo control } \\
(n=58)\end{array}$} & \multirow[b]{2}{*}{ Q3 } & \multicolumn{5}{|c|}{$\begin{array}{l}\text { Grupo experimental } \\
(n=46)\end{array}$} & \multicolumn{2}{|c|}{ U Mann Whitney } & \multirow[t]{2}{*}{$\mathbf{r}$} \\
\hline TMT & $\mathbf{M}$ & EE & $\mathrm{Me}$ & Q1 & & $\mathbf{M}$ & $\mathrm{EE}$ & $\mathrm{Me}$ & Q1 & Q3 & $U$ & $p$ & \\
\hline TMTA-Segundos & 30,3 & 1,5 & 28 & 23 & 30 & 30,4 & 1,1 & 28 & 24 & 30 & 1275,5 & 0,69 & 0,03 \\
\hline TMTA-Errores & 1,7 & 0,2 & 1 & 0 & 3 & 1,3 & 0,1 & 0 & 0 & 2,7 & 1082 & 0,07 & $-0,17$ \\
\hline TMTB-Segundos & 72,8 & 1,8 & 76 & 60 & 85 & 72,8 & 1,3 & 76 & 60 & 85 & 1321,5 & 0,93 & $-0,00$ \\
\hline TMTB-Errores & 4,5 & 0,3 & 5 & 2 & 7 & 4,5 & 0,2 & 5 & 2 & 7 & 1327 & 0,96 & 0,00 \\
\hline \multicolumn{14}{|l|}{ WCST } \\
\hline Categorías & 3,3 & 0,2 & 3 & 2 & 4 & 3,1 & 0,1 & 3 & 2 & 4 & 1172,5 & 0,28 & $-0,1$ \\
\hline Aciertos & 77,2 & 2,3 & 79 & 61 & 95 & 75,8 & 1,7 & 78 & 61 & 94,7 & 1200,5 & 0,38 & $-0,08$ \\
\hline Errores & 45,8 & 2,7 & 42 & 27 & 67 & 47,6 & 2,0 & 45,5 & 31,2 & 67 & 1146 & 0,21 & 0,12 \\
\hline Perseveraciones & 25,7 & 2,6 & 18 & 12 & 26 & 26,9 & 2,1 & 18 & 13,5 & 28 & 1315 & 0,9 & 0,01 \\
\hline \multicolumn{14}{|l|}{ STROOP } \\
\hline Palabra & 87,4 & 1,6 & 85 & 75 & 99 & 88,0 & 1,08 & 86,5 & 78 & 99 & 1312 & 0,88 & 0,01 \\
\hline Color & 79 & 1,6 & 80 & 72 & 88 & 79,8 & 1,2 & 80,5 & 72 & 88 & 1229 & 0,49 & 0,06 \\
\hline Palabra-color & 50 & 2,0 & 50 & 38 & 53 & 48,4 & 1,4 & 49 & 37 & 53 & 1097,5 & 0,12 & $-0,15$ \\
\hline Interferencia & 9,6 & 0,6 & 9,8 & $-2,3$ & $\begin{array}{l}14 \\
, 3\end{array}$ & 7,3 & 1,3 & 6,6 & $-4,0$ & 14,3 & 1008 & $0,03^{*}$ & $-0,20$ \\
\hline \multicolumn{14}{|l|}{ DÍGITOS } \\
\hline Dígitos directos & 7,2 & 0,2 & 6 & 6 & 8 & 7,45 & 0,14 & 8 & 6 & 8 & 1073 & 0,06 & 0,18 \\
\hline Número directos & 4,7 & 0,1 & 4 & 4 & 5 & 4,82 & 0,08 & 5 & 4 & 5 & 1113 & 0,12 & 0,15 \\
\hline Dígitos inversos & 6,0 & 0,1 & 6 & 6 & 6 & 6,36 & 0,14 & 6 & 6 & 8 & 954,5 & $0,006^{*}$ & 0,27 \\
\hline $\begin{array}{l}\text { Número } \\
\text { inversos }\end{array}$ & 3,9 & 0,1 & 4 & 4 & 4 & 4,1 & 0,08 & 4 & 4 & 5 & 862,5 & $0,000^{*}$ & 0,35 \\
\hline Total, Dígitos & 13,2 & 0,2 & 12 & 12 & 15 & 13,8 & 0,21 & 14 & 12 & 16 & 940,5 & $0,006^{*}$ & 0,26 \\
\hline Aritmética & 10,9 & 0,3 & 12 & 9,7 & 13 & 11,5 & 0,24 & 12 & 11 & 13 & 834,5 & $0,001^{*}$ & 0,32 \\
\hline $\begin{array}{l}\text { Letras y } \\
\text { números }\end{array}$ & 10,5 & 0,2 & 11 & 10 & 12 & 10,8 & 0,19 & 11 & 10 & 12 & 988 & $0,01^{*}$ & 0,23 \\
\hline \multicolumn{14}{|l|}{ FVF } \\
\hline Letra F & 10,4 & 0,2 & 11 & 9 & 12 & 10,6 & 0,42 & 11 & 9 & 12 & 1081 & 0,09 & 0,16 \\
\hline Letra A & 10,6 & 0,2 & 11 & 9 & 13 & 10,8 & 0,35 & 11 & 9,2 & 13 & 1151,5 & 0,22 & 0,11 \\
\hline Letra S & 10,7 & 0,2 & 11 & 8,2 & 13 & 10,8 & 0,41 & 11,5 & 8,2 & 13 & 1194,5 & 0,35 & 0,09 \\
\hline Letra M & 11,9 & 0,3 & 13 & 10 & 14 & 12,6 & 0,5 & 13 & 11 & 15 & 1014 & $0,03^{*}$ & 0,16 \\
\hline
\end{tabular}

Nota: M: Media, EE: Error Estándar, Me: Mediana, Q1: Cuartil 1, Q3: Cuartil 3. * $\mathrm{p}<, 05$, r: Tamaño efecto.

Fuente: Elaboración propia.

Para el análisis del rendimiento académico pretest y postest por cada uno de los grupos se empleó la prueba Wilcoxon, los resultados mostraron diferencias estadísticamente significativas para el grupo control con respecto al promedio académico $(Z=-3,4 ; p=0,001 ; r=0,44)$ presentando un mayor promedio en el postest, al igual que el grupo experimental $(Z=-5,78 ; p=0,000 ; r=0,85)$ el cual mostró un promedio académico mayor en el postest, es decir, después de la intervención con el programa ACTÍVATE+. 
¿El bajo rendimiento académico mejora a partir de...

Tabla 3

Puntuaciones de las pruebas neuropsicológicas postest grupos control y experimental

\begin{tabular}{|c|c|c|c|c|c|c|c|c|c|c|c|c|c|}
\hline \multirow{2}{*}{$\begin{array}{c}\text { Variable } \\
\text { TMT }\end{array}$} & \multicolumn{5}{|c|}{$\begin{array}{c}\text { Grupo control } \\
(n=58)\end{array}$} & \multicolumn{5}{|c|}{$\begin{array}{l}\text { Grupo experimental } \\
(n=46)\end{array}$} & \multicolumn{2}{|c|}{$\begin{array}{c}\text { U Mann } \\
\text { Whitney }\end{array}$} & \multirow[t]{2}{*}{$\mathbf{r}$} \\
\hline & M & EE & Me & Q1 & Q3 & M & $\mathrm{EE}$ & $\mathrm{Me}$ & Q1 & Q3 & $U$ & $p$ & \\
\hline TMTA-Segundos & 31,7 & 1,5 & 30 & 25 & 30,7 & 26,2 & 1,1 & 25 & 23 & 25 & 737 & $0,00^{*}$ & $-0,36$ \\
\hline TMTA-Errores & 0,6 & 0,1 & 0 & 0 & 1 & 0,07 & 0,03 & 0 & 0 & 0 & 812,5 & $0,00^{*}$ & $-0,40$ \\
\hline TMTB-Segundos & 73,6 & 2,3 & 75 & 60 & 85 & 72,3 & 1,8 & 75 & 65 & 80 & 272,5 & 0,68 & $-0,03$ \\
\hline TMTB-Errores & 2,9 & 0,2 & 3,5 & 0,7 & 5 & 0,1 & 0,05 & 0 & 0 & 0 & 70 & $0,00^{*}$ & $-0,62$ \\
\hline \multicolumn{14}{|l|}{ WCST } \\
\hline Categorías & 3,2 & 0,2 & 3 & 2 & 4 & 5,5 & 0,1 & 6 & 5 & 6 & 405,5 & $0,00^{*}$ & 0,58 \\
\hline Aciertos & 74,6 & 2,1 & 74 & 61 & 88,7 & 77,2 & 1,6 & 75 & 70 & 84,5 & 1217,5 & 0,44 & 0,07 \\
\hline Errores & 46,7 & 2,8 & 46 & 32 & 64 & 20,2 & 1,9 & 16 & 13 & 25 & 424 & $0,00^{*}$ & $-0,54$ \\
\hline Perseveraciones & 25,8 & 2,9 & 16 & 11,5 & 31 & 6,4 & 0,7 & 5 & 2 & 10,2 & 439,5 & $0,00^{*}$ & $-0,54$ \\
\hline \multicolumn{14}{|l|}{ STROOP } \\
\hline Palabra & 90,6 & 1,3 & 92 & 80 & 98 & 104, & 1,0 & 104 & 100 & 110,2 & 290 & $0,00^{*}$ & 0,63 \\
\hline Color & 81,2 & 1,6 & 84 & 72 & 92 & 98,6 & 2,7 & 108 & 88,5 & 112 & 508,5 & $0,00^{*}$ & 0,49 \\
\hline Palabra-color & 47,0 & 1,4 & 45 & 40 & 55 & 64,9 & 2,1 & 59 & 72 & 75 & 438,5 & $0,00^{*}$ & 0,54 \\
\hline Interferencia & 5,0 & 1,5 & 2,4 & $-3,5$ & 14,4 & 14,6 & 1,3 & 18 & 13,7 & 20 & 769 & $0,00^{*}$ & 0,34 \\
\hline \multicolumn{14}{|l|}{ DÍGITOS } \\
\hline Dígitos directos & 7,2 & 0,2 & 8 & 6 & 8 & 11,9 & 0,2 & 12 & 12 & 14 & 86 & $0,00^{*}$ & 0,77 \\
\hline Número directos & 4,6 & 0,1 & 5 & 4 & 5 & 7,09 & 0,1 & 7 & 7 & 8 & 119 & $0,00^{*}$ & 0,75 \\
\hline Dígitos inversos & 6,2 & 0,1 & 6 & 6 & 8 & 10,8 & 0,3 & 12 & 11,2 & 12 & 282 & $0,00^{*}$ & 0,65 \\
\hline $\begin{array}{l}\text { Número } \\
\text { inversos }\end{array}$ & 4,2 & 0,0 & 4 & 4 & 5 & 6,5 & 0,1 & 7 & 6,75 & 7 & 196,5 & $0,00^{*}$ & 0,71 \\
\hline Total, Dígitos & 13,4 & 0,3 & 14 & 12 & 16 & 22,8 & 0,5 & 24 & 22,5 & 26 & 103,5 & $0,00^{*}$ & 0,75 \\
\hline Aritmética & 10,7 & 0,3 & 12 & 9 & 12,2 & 16,2 & 0,5 & 18 & 14,7 & 19 & 388,5 & $0,00^{*}$ & 0,57 \\
\hline $\begin{array}{l}\text { Letras } \mathrm{y} \\
\text { números }\end{array}$ & 10,2 & 0,2 & 11 & 10 & 12 & 15,2 & 0,4 & 16 & 13,7 & 17 & 326,5 & $0,00^{*}$ & 0,61 \\
\hline \multicolumn{14}{|l|}{ FVF } \\
\hline Letra $F$ & 10,8 & 0,4 & 11 & 8 & 12,2 & 15,7 & 0,5 & 17 & 15,5 & 18 & 433 & $0,00^{*}$ & 0,54 \\
\hline Letra A & 11,6 & 0,3 & 12 & 10 & 14 & 17,0 & 0,5 & 19 & 17,2 & 20 & 389 & $0,00^{*}$ & 0,57 \\
\hline Letra S & 10,2 & 0,4 & 10 & 8 & 12,2 & 15,4 & 0,6 & 17 & 15 & 18 & 422 & $0,00^{*}$ & 0,55 \\
\hline Letra M & 10,7 & 0,3 & 11 & 9 & 13 & 16,8 & 0,5 & 18 & 16,7 & 19 & 282,5 & $0,00^{*}$ & 0,63 \\
\hline
\end{tabular}

Nota: M: Media, EE: Error Estándar, Me: Mediana, Q1: Cuartil 1, Q3: Cuartil 3. ${ }^{*} \mathrm{p}<, 05$, r: Tamaño efecto.

Fuente: Elaboración propia.

\section{Tabla 4}

Comparación del rendimiento académico grupos control y experimental

\begin{tabular}{|c|c|c|c|c|c|c|c|c|c|c|c|c|c|}
\hline \multirow{2}{*}{$\begin{array}{c}\text { Variable } \\
\begin{array}{c}\text { Rendimiento } \\
\text { académico }\end{array}\end{array}$} & \multicolumn{5}{|c|}{$\begin{array}{l}\text { Grupo control } \\
\quad(n=58)\end{array}$} & \multicolumn{5}{|c|}{$\begin{array}{c}\text { Grupo experimental } \\
(n=46)\end{array}$} & \multicolumn{2}{|c|}{$\begin{array}{l}\text { U Mann } \\
\text { Whitney }\end{array}$} & \multirow{2}{*}{$r$} \\
\hline & $\mathbf{M}$ & EE & Me & Q1 & Q3 & $\mathbf{M}$ & EE & Me & Q1 & Q3 & $U$ & p & \\
\hline Pretest & 3,23 & 0,02 & 3,3 & 3,2 & 3,4 & 3,26 & 0,01 & 3,3 & 3,2 & 3,4 & 1241,5 & 0,53 & - \\
\hline Postest & 3,32 & 0,02 & 3,4 & 3,2 & 3,5 & 3,89 & 0,05 & 3,9 & 3,7 & 4,2 & 302 & $0,00^{*}$ & 0,62 \\
\hline
\end{tabular}


Por otra parte, al comparar el rendimiento académico de los grupos control y experimental antes de la intervención no se observaron diferencias estadísticamente significativas $(p>0,05)$. Mientras que, al comparar el desempeño académico después de la aplicación del programa

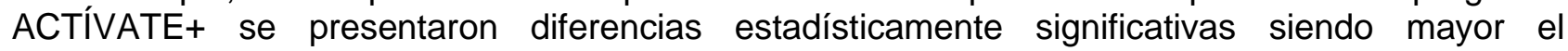
rendimiento académico en el grupo experimental $(p=0,000)$ (Tabla 4$)$. Este resultado muestra que el entrenamiento en FE puede tener un efecto positivo sobre el rendimiento académico de estudiantes con bajo desempeño escolar.

\section{Conclusiones y Discusión}

La deserción y la repetición escolar como consecuencia del bajo rendimiento académico son preocupaciones constantes en el ámbito educativo y de los profesionales que se ocupan de atender las necesidades de estudiantes, profesores y familiares, procurando brindar herramientas y estrategias pertinentes de intervención. Por tanto, la presente investigación tuvo como objetivo determinar la efectividad del programa ACTÍVATE+ en funciones ejecutivas diseñado para estudiantes con bajo rendimiento académico.

Según la revisión de Albarracín y Montoya (2016) sobre programas de intervención en estudiantes universitarios, así como la revisión de Pardos y González (2018) acerca de la intervención sobre las funciones ejecutivas (FE) desde el contexto educativo, no se han reportado estudios con programas similares al programa ACTíVATE+ que hayan sido aplicados específicamente a estudiantes entre los 13 y 19 años, siendo un aspecto innovador del presente trabajo y de relevancia como aporte al campo educativo desde la neuropsicología.

De acuerdo con los hallazgos sobre el desempeño de los estudiantes del grupo control pre y postest en las pruebas neuropsicológicas, se encontraron diferencias significativas en algunas tareas de las FE de Planeación, Memoria de Trabajo, Fluidez Verbal y Control Inhibitorio. Esto puede asociarse al desarrollo de las sesiones de apoyo psicopedagógico por parte de los participantes del grupo control, enfocadas a optimizar procesos de aprendizaje para mejorar los hábitos y técnicas de estudio, como la planificación de actividades y administración del tiempo además de asesorías psicológicas. Esto demuestra que dichas asesorías pueden ser útiles para optimizar algunas FE relacionadas con la atención sostenida y dividida, memoria de trabajo, fluidez verbal y control inhibitorio. De la misma manera, es coherente con los estudios revisados por Albarracín y Montoya (2016) donde los programas de autorregulación, tutorías y habilidades académicas en general aportan a la planificación, el monitoreo de aspectos afectivomotivacionales, la comprensión lectora y la escritura.

Con respecto al grupo experimental, se encontraron diferencias significativas en la mayoría de los dominios de FE evaluados mostrando que el programa ACTíVATE+ favoreció el desempeño ejecutivo de los estudiantes en más dominios en comparación con los participantes del grupo control que recibieron solo el apoyo psicopedagógico. Estos hallazgos son coherentes con lo reportado por otros investigadores que han empleado programas online o computarizados para mejorar FE como la planeación en niños escolares (Ríos et al., 2014), la flexibilidad mental y planeación en estudiantes con discapacidad intelectual (De La Torre et al., 2017) y la velocidad de procesamiento, la memoria de trabajo, la atención ejecutiva, el control inhibitorio y la flexibilidad cognitiva en estudiantes universitarios utilizando el programa ACTÍVATE+ (Filippelli, 2018). 
Un aspecto relevante, es que los resultados pueden no ser atribuibles a la curva de aprendizaje de las pruebas neuropsicológicas, dado que se emplearon versiones alternas para el postest y las tareas del programa ACTÍVATE+ fueron desarrolladas a partir de los paradigmas de entrenamiento de $\mathrm{FE}$, siendo cuidadosamente diseñadas para que no presentaran estímulos o procedimientos exactamente iguales a los de las pruebas aplicadas, además, al estar en formato computarizado es una manera distinta de presentar las tareas al empleado en lápiz y papel, con excepción del WCST.

De igual forma, al comparar los grupos control y experimental en el desempeño de las FE antes de la intervención, se encontró que el grupo control tuvo un menor desempeño en Control Inhibitorio (Interferencia), Memoria de Trabajo (Dígitos Inversos, Número Inversos, Total Dígitos, Aritmética y, Letras y Números) y en Fluidez Verbal (Letra M) al compararlo con el grupo experimental, el cual mostró un mejor rendimiento en las pruebas neuropsicológicas antes de iniciar la intervención, lo que pudo ser una ventaja en el posterior desempeño de las pruebas; sin embargo, los tamaños del efecto se encontraron en un rango de medio a bajo.

Igualmente se observó que el grupo experimental tuvo un mejor desempeño en todos los dominios de FE después de la intervención, con tamaños del efecto de medios a grandes comparado con el grupo control.

Por consiguiente, el grupo experimental tuvo un mejor rendimiento en las pruebas de la función Planeación (excepto en TMTB-Segundos y WCST Aciertos). Por tanto, los participantes demostraron mejor desempeño en procesos de atención, seguimiento visual, velocidad psicomotora (TMT-A), capacidad de planeación y abstracción, siendo funciones importantes para la consecución de objetivos y metas (Tsukiura et al., 2001) que median en los procesos de aprendizaje para la organización del estudio, las tareas de clase y gestión del tiempo (Pardos y González, 2018). Con respecto al dominio de Control conductual o inhibitorio, el grupo experimental también presentó un mejor desempeño en la atención selectiva, la inhibición cognitiva de respuestas automáticas, supresión de impulsos inapropiados, pensamientos y acciones, favoreciendo el análisis y elección de la mejor respuesta o procedimiento para dar solución a un problema o tarea (Flores et al., 2014). En cuanto a la Memoria de Trabajo, el grupo experimental mostró un rendimiento mayor en los procesos de atención, memoria y procesamiento auditivo a corto plazo, secuenciación, concentración, capacidad de aprendizaje, memoria a largo plazo, alerta mental, transformación de la información e imaginación visoespacial, fundamentales en los procesos de toma de decisiones, aprendizaje, razonamiento y planeación (Baddeley, 1999, Funahashi, 2006); así como para el seguimiento de instrucciones, procesos de lecto-escritura y cálculo mental (Pardos y González, 2018). Por último, en el dominio de Fluidez Verbal, los participantes del grupo experimental evidenciaron un mejor desempeño en las estrategias de búsqueda de información, retención de información lingüística y velocidad, en la habilidad para decodificar y codificar tareas verbales, las cuales favorecen mejores resultados en el rendimiento académico (Rojas y Rincón, 2015).

En consecuencia, se demuestra que el programa ACTÍVATE+ permitió la optimización de las FE de una forma más significativa en el grupo experimental, un factor relacionado pudo ser que la muestra tenía como característica no presentar trastornos a nivel cognitivo o psiquiátrico, por tanto, las funciones cerebrales de los participantes estaban dentro de lo que se consideraría la normalidad y al estar en la etapa de la adolescencia presentan cambios cerebrales representativos que inciden positivamente en el aprendizaje, procesos que corresponden con la mielinización, cambios en la sustancia gris y blanca que favorecen la velocidad del procesamiento de la información, cambios en estructuras como el estriado ventral, el cuerpo calloso y la glándula pineal asociados a habilidades como el análisis, la resolución de problemas y la toma de decisiones; así como el aumento y maduración del cerebelo y de la corteza frontal relacionadas con las FE y procesos cognitivos superiores (Martín y Rodríguez, 2015; Tamayo et al., 2018). Igualmente, los resultados demuestran que el entrenamiento de procesos ejecutivos en períodos 
de escolarización logra aumentar el desempeño de las FE en población adolescente (García, González et al., 2014, Flores et al., 2014).

Por otro lado, al comparar el rendimiento académico entre los grupos control y experimental, se encontró que ambos mostraron un mayor rendimiento académico después de la fase de intervención, sugiriendo que las intervenciones fueron efectivas influyendo positivamente en el desempeño académico de los estudiantes. Lo anterior es coherente con el trabajo de Albarracín y Montoya (2016) donde se concluye que las intervenciones psicopedagógicas pueden ser útiles para la mejora de estrategias cognitivas que posteriormente influirán en el rendimiento académico, lo cual fue evidenciado en los resultados del grupo control asociados al apoyo psicopedagógico. No obstante, al analizar el tamaño del efecto se encuentra que las diferencias en el grupo control fueron moderadas y el porcentaje de mejora estuvo en un 1,8\% aproximadamente; mientras que en el grupo experimental fueron altas con un porcentaje de mejora de aproximadamente $12,6 \%$, indicando que los resultados en el grupo experimental son más generalizables y significativos, y que pueden ser atribuibles al efecto que tiene el programa ACTíVATE+ sobre el rendimiento académico. En este sentido, se puede considerar que el programa ACTÍVATE+ muestra validez ecológica, dado que potencia las habilidades cognitivas de los sujetos, implicadas en el funcionamiento cotidiano de su vida escolar (García, Tirapu et al., 2007), contribuyendo al incremento del desempeño académico evidenciado en la aprobación del periodo académico.

Así mismo, este resultado es afín con lo expuesto por Betancur et al. (2016) quienes demostraron mejora en las FE de control inhibitorio, fluidez y planeación, y el rendimiento académico después del entrenamiento cognitivo en un niño de 10 años con desarrollo normal esperado según la edad. Igualmente, De la Torre et al. (2017) mostraron diferencias en los componentes de planeación y flexibilidad al emplear el programa computarizado Lumosity en niños entre los 7 y 12 años con discapacidad intelectual, donde el grupo caso tuvo mejor desempeño que el grupo control en ambos componentes, considerados relevantes en los procesos educativos y adaptativos. Asimismo, el estudio de Filippelli (2018) donde se empleó el programa ACTÍVATE+ en 60 estudiantes (30 grupo control y 30 grupo experimental) entre los 17 y 29 años de primer año de psicología, mostró mejora en velocidad de procesamiento, memoria de trabajo, atención ejecutiva, control inhibitorio y flexibilidad cognitiva en el grupo experimental, concluyendo que el programa ACTIVATE+ abre la posibilidad de una nueva herramienta para estudiantes que presenten algún tipo de dificultad en sus FE o que deseen continuar desarrollándolas.

Estos hallazgos demuestran que el programa ACTíVATE+ es una herramienta efectiva en el entrenamiento de las FE para mejorar tanto estas funciones como el rendimiento escolar de estudiantes con bajo desempeño académico, como lo expresan Jiménez y Marques (2018) las intervenciones en FE proporcionan apoyo y estimulación para la formación y maduración de los dominios del sistema ejecutivo, lo que es fundamental para mejorar el rendimiento escolar. Igualmente, se demuestra que el enfoque de restauración desde el cual se diseñó el programa ACTÍVATE + fue útil en la mejora de las FE a partir de la estimulación cognitiva de los estudiantes en condiciones de normalidad, a diferencia de los programas de rehabilitación neuropsicológica que se enfocan más en la recuperación de las funciones originadas por un daño o pérdida (Ginarte-Arias, 2002).

El programa ACTíVATE+ al igual que otros programas como Lumosity y Portal 2 que evidencian mejoras en la resolución de problemas (Shute et al., 2015), muestra su potencial en la optimización de los procesos de planeación, fluidez verbal, memoria de trabajo y control inhibitorio fundamentales para el desarrollo de tareas, toma de decisiones, organización, control de impulsos, procesos de lectura, escritura y cálculo mental impactando positivamente en el desempeño escolar. Por tanto, el programa ACTíVATE+ puede ser empleado en contextos educativos formales y no formales como complemento y apoyo a los programas psicopedagógicos orientados por los diferentes profesionales y dirigidos a estudiantes con bajo rendimiento académico con el fin de contribuir a la disminución de la deserción y repetición escolar. Una de las 
ventajas del programa corresponde con su utilización vía online estableciéndose como una herramienta pertinente en cuanto a su empleo, accesibilidad y usabilidad desde la virtualidad en el contexto educativo; y aunque en algunos entornos puede ser una limitante, el crecimiento exponencial del acceso a internet a nivel mundial exige soluciones que se ajusten a los cambios sociales en los procesos de transformación digital que atraviesan las instituciones educativas en una sociedad donde las tecnologías digitales aparecen como las formas ideales para comunicarse, relacionarse, organizarse, administrarse e intervenir las situaciones problemáticas.

Según Núñez et al. (2011) el generar programas en formato computarizado puede lograr mayor motivación en los participantes, aunque este estudio no midió directamente la motivación en los participantes, los mismos expresaron agrado e interés por las tareas realizadas, siendo un aspecto que puede ser evaluado en posteriores investigaciones. Asimismo, la facilidad y precisión en el registro de los datos proponen al programa ACTÍVATE + como una herramienta consistente y fiable (De la Fuente y Hernández, 2014). Una limitación del programa es que todavía no es funcional para teléfonos inteligentes lo que restringe su utilización.

Finalmente, se proyecta poder realizar diferentes mediciones después de la aplicación del programa con el fin de verificar el efecto en el tiempo sobre las FE y el rendimiento académico consolidando su validez ecológica. Igualmente, se pretende seguir la revisión minuciosa del programa con el fin que sea cada vez más idóneo para investigadores y usuarios considerando la posibilidad de ampliar su utilización en otras poblaciones como las clínicas.

\section{Referencias bibliográficas}

Albarracín, A. y Montoya, D. (2016). Programas de intervención para estudiantes universitarios con bajo rendimiento académico. Informes Psicológicos, 16(1), 13-34.

Álvarez, G. (2012). Las nuevas tecnologías en el contexto universitario: Sobre el uso de blogs para desarrollar las habilidades de lectoescritura de los estudiantes. Revista de $\begin{array}{lllll}\text { Universidad y Sociedad del Conocimiento, } & \text { 9(2), }\end{array}$ http://dx.doi.org/10.7238/rusc.v9i2.1160

Arán-Filippetti, V. y Richaud de Minzi, M. (2011). Efectos de un programa de intervención para aumentar la reflexividad y la planificación en un ámbito escolar de alto riesgo por pobreza. Universitas Psychologica, 10(2), 341-354. https://doi.org/10.11144/Javeriana.upsy10-2.epia

Arango, J. y Rivera, D. (2015). Neuropsicología en Colombia: Datos normativos, estado actual y retos a futuro. Universidad Autónoma de México.

Arango, J., Rivera, D. y Olabarrieta, L. (2017). Neuropsicología infantil. Manual Moderno.

Baddeley, A. (1999). Memoria humana. Teoría y práctica. McGraw-Hill.

Bahamón, M. y Reyes, R. (2014). Caracterización de la capacidad intelectual, factores sociodemográficos y académicos de estudiantes con alto y bajo desempeño en los 
exámenes Saber Pro año 2012. Avances en Psicología Latinoamericana, 32(3), 459-476. http://dx.doi.org/10.12804/apl32.03.2014.01

Blanco, M. L., Chacón, J. E., Contreras, A. T., Corredor, M. F., Martínez, J., Muñoz, A. L., Palencia, V. V., Penagos, L. A. M., Ríos, S. G. y Zapata, M. F. (2020). Alcances y limitaciones de programas de entrenamiento cognitivo computarizado dirigidos al trastorno por déficit de atención con hiperactividad. Revista Enfoques, 3(1), 91-105. http://200.21.15.145/index.php/EFQ/article/view/290/533

Barceló, E., Lewis, S. y Moreno, M. (2006). Funciones ejecutivas en estudiantes universitarios que presentan bajo y alto rendimiento académico. Psicología desde el Caribe, 18, 109-138.

Benítez, M., Barajas, J. y Hernández, I. (2014). Efecto de la aplicación de una estrategia de comprensión de lectura en un entorno virtual. Revista Electrónica de Investigación Educativa, 16(3), 71-87. http://redie.uabc.mx/vol16no3/contenido-benitezbh.html

Bernal-Ruiz, F., Rodríguez-Vera, M. y Ortega, A. (2020). Estimulación de las funciones ejecutivas y su influencia en el rendimiento académico en escolares de primero básico. Interdisciplinaria, 37(1). https://doi.org/10.16888/interd.2020.37.1.6

Betancur, M., Molina, D. y Cañizales, L. (2016). Entrenamiento cognitivo de las funciones ejecutivas en la edad escolar. Revista Latinoamericana de Ciencias Sociales, Niñez y Juventud, 14(1), 359-368. http://dx.doi.org/10.11600/1692715x.14124160615

Borkowski, J., Benton, A. y Spreen, O. (1967). Word fluency and brain damage. Neuropsychologia, 5(2), 135-140. https://doi.org/10.1016/0028-3932(67)90015-2

Cabero, J. (2010). Los retos de la integración de las TICs en los procesos educativos. Límites y posibilidades. Perspectiva Educacional, 49(1), 32-61.

Carbonero, M., Román, J. y Ferrer, M. (2013). Programa para "aprender estratégicamente" con estudiantes universitarios: Diseño y validación experimental. Anales de Psicología, 29(3), 876-885. http://dx.doi.org/10.6018/analesps.29.3.165671

De la Fuente, Y. y Hernández, J. (2014). Las tecnologías de la información y la comunicación como entorno de convergencia tecnológica. El Design Thinking aplicado a la discapacidad intelectual. Revista Internacional de Sociología, 72(Extra_1), 93-112. https://doi.org/10.3989/ris.2013.01.11

De La Torre, D., Galvis, A., Lopera, A. y Montoya, D. (2017). Función ejecutiva y entrenamiento computarizado en niños de 7 a 12 años con discapacidad intelectual. Revista Chilena de Neuropsicología, 12(2), 14-19.

Ferrando, L., Bobes, J., Gibert, J., Soto, M. y Soto, O. (2000). MINI Entrevista Neuropsiquiátrica Internacional, versión en español. https://www.academia.cat/files/425-7297DOCUMENT/MinientrevistaNeuropsiquatribalnternacional.pdf

Filippelli, F. (2018). Entrenamiento de funciones ejecutivas en estudiantes de primer año de psicología de la Universidad Favaloro [Tesis de pregrado no publicada]. Universidad Favaloro.

Flores, J., Castillo, R. y Jiménez, N. (2014). Desarrollo de funciones ejecutivas, de la niñez a la juventud. Anales de Psicología, 30(2), 463-473. http://dx.doi.org/10.6018/analesps.30.2.155471 
Fortuny, L. y Heaton, R. (1996). Standard versus computerized administration of the Wisconsin Card Sorting Test. The Clinical Neuropsychologist, 10(4), 419-424. https://doi.org/10.1080/13854049608406702

Funahashi, S. (2006). Prefrontal cortex and working memory processes. Neuroscience, 139, 251261. https://doi.org/10.1016/j.neuroscience.2005.07.003

García, T., González, P., Areces, D., Cueli, M. y Pérez, C. (2014). Funciones ejecutivas en niños y adolescentes: Implicaciones del tipo de medidas de evaluación empleadas para su validez en contextos clínico y educativos. Papeles del Psicólogo, 35(3), 215-223.

García, T., Rodríguez, C., González-Castro, P., Álvarez, D., Cueli, M. y González-Pienda, J. (2013). Funciones ejecutivas en niños y adolescentes con trastorno por déficit de atención con hiperactividad y dificultades lectoras. International Journal of Psychology and Psychological Therapy, 13(2), 179-194.

García, A., Tirapu, J. y Roig, T. (2007). Validez ecológica en la exploración de las funciones ejecutivas. Anales de Psicología, 23(2), 289-299.

Ginarte-Arias, Y. (2002). Rehabilitación cognitiva. Aspectos teóricos y metodológicos. Revista de Neurología, 34(9), 870-876. https://doi.org/10.33588/rn.3509.2002418

Golden, C.J. (2010). Manual Stroop: Test de colores y palabras. TEA Ediciones S.A.

Holmes, J. y Gathercole, S. (2014). Taking working memory training from the laboratory into schools. Educational Psychology, 34(4), 440-450. https://doi.org/10.1080/01443410.2013.797338

Jiménez, S. y Marques, D. (2018). Impacto de la intervención neuropsicológica infantil en el desarrollo del sistema ejecutivo. Análisis de un caso. Avances en Psicología Latinoamericana, 36(1), 11-28.

http:// dx.doi.org/10.12804/revistas.urosario.edu.co/apl/a.4150

Kaufman, A. y Kaufman, N. (1997). Test Breve de Inteligencia de Kaufman. Pearson.

Knouse, L., Feldman, G. y Blevins, E. (2014). Executive functioning difficulties as predictors of academic performance: Examining the role of grade goals. Learning and Individual Differences, 36, 19-26. https://doi.org/10.1016/j.lindif.2014.07.001

Korhonen, J., Linnanmäki, K. y Aunio, P. (2014). Learning difficulties, academic well-being, and educational dropout: A person-centered approach. Learning and Individual Differences, 31(2), 1-10. https://doi.org/10.1016/j.lindif.2013.12.011

Lezak, M. (1995). Neuropsychologycal assesment. Oxford University Press.

Lubrini, G., Periáñez, J. y Ríos, M. (2009). Introducción a la estimulación cognitiva y la rehabilitación neuropsicológica. En E. Muñoz Marrón (Ed.), Estimulación cognitiva y rehabilitación neuropsicológica (pp. 13-32). Editorial UOC.

Martín, P. y Rodríguez, A. (2015). La intervención desde la base neuropsicológica y metodologías que favorecen el rendimiento escolar. En Ministerio de Educación, Cultura y Deporte de España (Ed.), Madrid: Procesos y programas de neuropsicología educativa (pp. 14-32). Secretaría General Técnica. 
Ministerio de Educación Nacional [MEN]. (2009). Deserción estudiantil en la educación superior colombiana: Metodología de seguimiento, diagnóstico y elementos para su prevención. Ministerio de Educación Nacional.

Miñaca, M. y Hervás, M. (2013). Intervenciones dirigidas a la prevención y abandono del fracaso escolar: Un estudio de revisión. Revista Española de Educación Comparada, 21, 203220. https://doi.org/10.5944/reec.21.2013.7620

Montero, I. y León, O. (2005). Sistema de clasificación del método en los informes de investigación en Psicología. International Journal of Clinical and Health Psychology, 5(1), 115-127.

Montes, M., Flores, R. y Roqueta, C. (2020). Revisión sistemática del efecto de las funciones ejecutivas en el rendimiento académico. Ágora de Salut, 7, 205-215. http://dx.doi.org/10.6035/AgoraSalut.2020.7.21

Montoya, D., Aguirre, D., Díaz, C. y Pineda, D. (2018). Executive functions and high intellectual capacity in school-age: Completely overlap? International Journal of Psychological Research, 11(1), 19-32. http://dx.doi.org/10.21500/20112084.3239

Núñez, J., Cerezo, R., González-Pienda, J., Rosário, P. y Valle, A. (2011). Implementation of training programs in self-regulated learning strategies in Moodle format: Results of an experience in higher education. Psicothema, 23(2), 274-281.

Organización de Estados Iberoamericanos [OEI]. (2010). Metas educativas 2021: La educación que queremos para la generación de los bicentenarios. Organización de Estados Iberoamericanos para la Educación, la Ciencia y la Cultura.

Pardos, A. y González, M. (2018). Intervención sobre las funciones ejecutivas (FE) desde el contexto educativo. Revista Iberoamericana de Educación, 78(1), 27-42. https://doi.org/10.35362/rie7813269

Pérez, J. (2015). Desarrollo de un programa de intervención neuropsicológica para la mejora del rendimiento escolar en estudiantes de primaria colombianos [Tesis de Maestría, Universidad Internacional de la Rioja]. https://reunir.unir.net/handle/123456789/3720

Pineda, D. (2000). La función ejecutiva y sus trastornos. Revista de Neurología, 30(8), 764-768.

Portellano, J. y García, J. (2014). Neuropsicología de la atención, las funciones ejecutivas y la memoria. Editorial Síntesis.

Ríos, S., Bolívar, H., García, K. y Montoya, C. (2014). Estudio piloto de un prototipo inmersivo online de entrenamiento cognitivo para la planificación en niños. Revista Vanguardia Psicológica, 5(1), 22-31.

Rodríguez, N. (2016). Funciones ejecutivas en estudiantes con alto y bajo nivel de desempeño académico [Tesis de Maestría, Universidad Internacional de la Rioja]. http://reunir.unir.net/handle/123456789/4585

Roebers, C., Cimeli, P., Röthlisberger, M. y Neuenschwander, R. (2012). Executive functioning, metacognition, and self-perceived competence in elementary school children: An explorative study on their interrelations and their role for school achievement. Metacognition Learning, 7, 151-173. https://doi.org/10.1007/s11409-012-9089-9 
Rojas, J.A. y Rincón, C.F. (2015). Estudio descriptivo comparativo de las funciones ejecutivas frías y rendimiento académico en adolescentes. Revista Mexicana de Neurociencias, 16(1), 40-50.

Sánchez, F., Velasco, T., Ayala, M. y Pulido, X. (2016). Trayectorias de permanencia, deserción y repitencia en la educación secundaria colombiana y sus factores asociados. Ministerio de Educación Nacional. https://papers.ssrn.com/sol3/papers.cfm?abstract id=2927172

Shehan, D., Shytle, D., Milo, K., Lecrubier, Y. y Jergueta, T. (2006). Mini International Neuropsychiatric Interview. For children and adolescents (M.I.N.I.-KID). Mini entrevista neuropsiquiátrica internacional para niños y adolescentes M.I.N.I.-(N-A). Adaptación al español colombiano. Pineda, D. Universidad de San Buenaventura-Universidad de Antioquia.

Shute, V., Ventura, M. y Ke, F. (2015). The power of play: The effects of Portal 2 and Lumosity on cognitive and noncognitive skills. Computers \& Education, 80, 58-67. https://doi.org/10.1016/i.compedu.2014.08.013

Sistema para la Prevención de la Deserción en la Educación Superior [SPADIES]. (2017). Deserción estudiantil. https://www.mineducacion.gov.co/sistemasdeinformacion/1735/w3article-212299.html? noredirect=1

Spreen, E. y Strauss, O. (1998). Compendium of neuropsychological test. Oxford University.

Tamayo, L., Merchán M., Hernández C., Ramírez B. y Gallo R. (2018). Nivel de desarrollo de las funciones ejecutivas en estudiantes adolescentes de los colegios públicos de EnvigadoColombia. Revista CES Psicología, 11(2), 21-36. http://dx.doi.org/10.21615/ cesp.11.2.3

Tombaugh, T. (2004). Trail Making Test A and B: Normative data stratified by age and education. Archives of Clinical Neuropsychology, 19, 203-214. https://doi.org/10.1016/S08876177(03)00039-8

Torres, J., Acevedo, D. y Gallo, L. (2015). Causas y consecuencias de la deserción y repitencia escolar: Una visión general en el contexto latinoamericano. Cultura Educación y Sociedad, 6(2), 157-187.

Tsukiura, T., Fujii, T., Takahashi, T., Xiao, R., Inase, M., lijima, T., Yamadori, A., y Okuda, J. (2001). Neuroanatomical discrimination between manipulating and maintaining processes involved in verbal working memory: a functional MRI study. Cognitive Brain Research, 11, 13-21. https://doi.org/10.1016/S0926-6410(00)00059-8

Wechsler, D. (2002). WAIS III. Test de Inteligencia para adultos. Paidós.

Wechsler, D. (2005). WISC IV. Test de Inteligencia para niños. TEA Ediciones.

Fecha de entrada: 29 junio 2020

Fecha de revisión: 2 noviembre 2020

Fecha de aceptación: 23 diciembre 2020 